Session 2648

\title{
Autonomous Ground Robotic Vehicles for Electrical Engineering Technology
}

\author{
Robert N. Riggins, Bruce V. Mutter \\ Bluefield State College \\ bmutter@bluefieldstate.edu
}

\begin{abstract}
This paper discusses the design and provides an analysis of the performance of an autonomous ground robotic vehicle (GRV) called Vasilius. Three sections of focus are presented. First, the paper describes the hardware and software design of Vasilius. A novel idea of modeling an autonomous vehicle after human senses and the human decision-making process is presented. For example, Vasilius integrates a large number of independent sensors such as stereoscopic vision to incorporate short-range reaction as well as long-range planning. In addition, the GRV uses two computers in a decentralized control scheme.

Secondly, the paper reports the performance of Vasilius, relating theoretical predictions to actual behavior. Performance measures include object avoidance, lane following, waypoint accuracy, follow-the-leader, ramp climbing, turn reaction time, battery life, stop reaction time, behavior in dead-ends and traps, pothole avoidance, and safety. Finally, the paper discusses current on-going research topics with Vasilius, including the incorporation of Kalman Filtering, mapping, and learning. The important side benefit of implementing this applied research robotics project in the ELET 492 Senior Design course, where student teams build robotics for our defense agency clients, is realized with their success in the annual International Ground Robotics Vehicle Competition. This program has proven to be a definite benefit to motivating our students as well as satisfying our clients.
\end{abstract}

\section{Introduction}

The 2002-2003 Vasilius Team of Bluefield State College was honored to bring new and innovative ideas to the 11 th Annual Intelligent Ground Vehicle Competition (IGVC). The vehicle draws upon many features of past GRV designs. For example, Vasilius uses a camera vision system and a laser measurement system like most GRVs. However, in addition to these standard systems, we decided to explore a new idea of modeling an autonomous vehicle after human senses and the human decision-making process. First, the vision system of the vehicle has stereoscopic vision capability, mimicking a human's eyes. Stereoscopic vision allows Vasilius to create a 3D map from cameras alone. Second, we prioritized various sensor inputs used by the autonomous algorithm. This simulates a human making a decision and slightly later in time changing that decision because of an unforeseen problem. The student team developed a winning vehicle that competed in all four challenges of the 2003 competition: the Autonomous Challenge, Vehicle Design, Navigation Challenge, and Follow-The-Leader Challenge. In this report, we describe the development of Vasilius and how we incorporated the "human" theme throughout the design. This report is divided into sections of focus. The sections of focus are: Design 
Process, Mechanical System, Electrical System, Software Design, Analysis/Predicted Performance, and Other Design Considerations.

\section{Design Process}

To achieve creative and innovative solutions to any engineering project requires both a systematic and organized design process. In the initial meeting of ELET 492 in the fall of 2002, our GRV student team sought to produce a robot which would satisfactorily perform its required functions while staying within our budget. The team decided that the design process on 'Vasilius' would have the following six phases: identification of the customer, problem recognition, preliminary ideas, problem solution, construction, and testing, as shown in Figure 1.

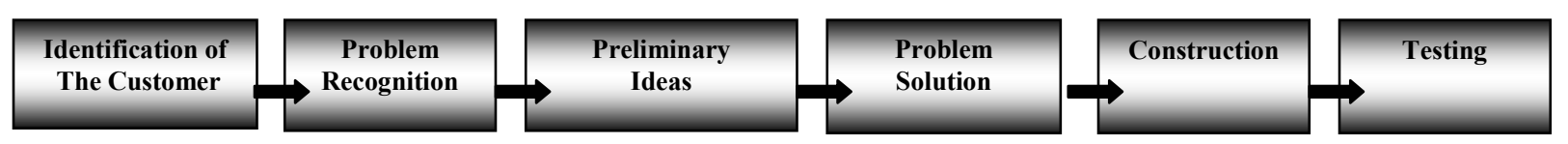

\section{Figure 1: Design Process}

\subsection{Identification of Customer}

The robot design began with the identification of our customers. The students decided for their purposes that the customers were the students, BSC, our community and sponsors, and the IGVC judges. The GRV team identified talents among team members in a wide array of disciplines and would most likely produce a robot that was pleasing to our college and community. Local companies donated many of the parts used on the robot. The instructor emphasized the importance of showing our sponsors that our finished product is a success. Therefore, students document progress on a weekly basis and give presentations to the College and sponsors once a semester.

\subsection{Problem Recognition}

Recognizing the problem and setting the overall goal for the project constituted the next phase of the design process. The goal was straightforward - we wanted to win all four competitions in the summer of 2003. The team studied the requirements from the 2002 competition and met to discuss ideas. Few engineering problems have clear-cut methods from start to finish but rather evolve over the duration of the design process. In recognizing this inevitability, our Autonomous GRV (AGRV) team initially made a list of the requirements, limitations, and general factors affecting our ultimate goal.

\subsection{Preliminary Ideas}

Next in the design process was to brainstorm preliminary ideas so as to profit from a collective interchange of ideas rather than just one single idea. It is in this stage of the design process that rough sketches are drawn with preliminary notes. For instance, our team desired to stray away from the bulkiness exhibited by "Centurion", the BSC AGRV entry from last year, with the hope that a more compact robot would enable us to maneuver in and around obstacles more effectively. The team thought it was advantageous to explore as many design possibilities as possible. The team then selected the best idea and made detailed drawings using Computer Aided Drafting techniques enabling us to determine sizes, angles, clearances, and other 
information necessary for further analysis. After deciding on a plan for our new GRV, construction would begin with extensive testing throughout the process so as to produce the best robot at competition.

\subsubsection{Team Organization}

During the preliminary ideas phase of the design process, the students had input in organizing the team. The Vasilius Team consists of eight engineering technology students. All of the Vasilius team members are undergraduates from various disciplines, including electrical, mechanical, computer, civil and architectural engineering technology. All team members participated in the initial design process. We assigned each member tasks that matched their abilities. Our team devoted approximately 1600 person hours in the development, design, fabrication and testing of this project. The team members for Vasilius are identified in Table 1.

\begin{tabular}{|l|l|l|}
\hline Team Members & \multicolumn{1}{|c|}{ Responsibilities } & \multicolumn{1}{|c|}{ Level- Major } \\
\hline Jarrod Snider & $\begin{array}{l}\text { Project Leader, Programmer, } \\
\text { Structure, Testing, Electronics, } \\
\text { GPS, LMS, Vision, Networking }\end{array}$ & Junior -Electrical \\
\hline Amy Snider & $\begin{array}{l}\text { Electronics, Testing, Vision, } \\
\text { Structure, Fabrication }\end{array}$ & Junior-Electrical \\
\hline Jeremy Woody & Electronics & Senior-Electrical \\
\hline Ravi Srivastava & LMS, Programmer & Senior-Electrical \\
\hline Donnie Walker & Structure, Electronics & Junior-Electrical \\
\hline Steve Paisley & Structure, Power Systems & Junior-Electrical \\
\hline Jack Lamm & Structure, Fabrication & Senior-Electrical \\
\hline Kenny Dunford & Computer-Aided Design & Senior- Architectural \\
\hline
\end{tabular}

Table 1: 2002-2003 Team Members

\subsubsection{Design Tools}

The mechanical design of Vasilius was accomplished using various software packages including AutoCAD, Inventor, and Rhino. For the electrical system design we used Electronic Workbench. After identifying our target specifications from our initial meetings concerning how the robot should look and perform, several students outside the team produced initial conceptual drawings using their respective software packages. 


\subsection{Problem Solution}

Once the lists of drawings were developed, the GRV team chose one that adequately met the demands imposed by the competition. By taking a systematic approach throughout vehicle development, the team was able to create a product that was accessible, well-organized, and compact. The preliminary designs on the computer-aided design packages minimized costly fabrication errors. The most important approach in any robotic vehicle design is to plan exactly how all the parts will coordinate with each other. This process led to the discussion of ideas on system integration.

\subsubsection{System Integration}

In developing the GRV solution, our GRV team placed emphasis on system integration because of the necessity of coordination between many distinct units in the robot. We instituted two concepts for system integration for Vasilius. One is a decentralized control concept and the other is a planning/reactive concept. Both concepts mimic human behavior.

\subsubsection{Decentralized Control Concept}

Vasilius is controlled by two computer systems. One computer is dedicated to vision and planning functions. The other computer controls reaction sensors and sudden changes in the long-range plan. Control is thus divided between these two computers.

\subsubsection{Planning/Reactive Concept}

The Vasilius program integrates the inputs from the five sensors in order to perform the major functions of the robot: lane following, obstacle avoidance, leader following, and waypoint navigation. As described in Section 5 on Software Design, these sensors are divided into planning sensors and reactive sensors. The reactive sensors have a higher priority.

\subsection{Construction}

Construction of Vasilius required manufacturing and engineering skills to work hand-in-hand. An initial design was given to the manufacturing team. The design had to be modified to accommodate the manufacturing process as well as the team's abilities. A final compromise was reached such that the overall design would only slightly deviate from the initial design. These slight deviations included component placement, amount of steel used, and weight.

\subsection{Testing}

Again, the three basic robotic topics were in order: mechanics, control, and sensor design. Mechanics as utilized on Vasilius included the design and structure of our autonomous robot. Control includes the electrical and mechanical systems, task planning, and implementation including both hardware and software. Sensor design includes the design of sensors and sensor systems and algorithms for sensory data acquisition and analysis. 


\subsection{Senior Design Course Integration}

The structure of the senior design course in which the student team designed and built the Vasilius GRV is described in Section 8.

\section{Mechanical System}

The overall mechanical design of Vasilius focused on simplicity, durability, compactness, maintainability and, most importantly, safety. The Vasilius team was able to meet every aspect of design. With the optimal mechanical design of Vasilius, the team produced an excellent platform for the vehicle. The mechanical design was divided into three separate categories: vehicle frame, drive system, and vehicle body.

\subsection{Vehicle Frame}

The vehicle frame is constructed of steel tubing. Steel tubing was chosen due to its light weight, durability, and its ability to house wiring. The tubing acts as a conduit to conceal and organize connections as well as shielding vulnerable lines from RF noise. Two types of $1 / 8$ inch thick tubing were used. 1 inch square tubing was used for the parts of the frame that did not require significant drilled holes. This allowed the design team to keep the weight to a minimum. The second type of tubing was $1.5 \times 1$ inch and was used for the remainder of the vehicle frame. The tubing was welded together in a simple rectangular arrangement. The rectangular design allowed the frame to be very strong while creating a protective carriage that housed the batteries, chargers, and other various components. A 1/16 inch thick steel plate was used on the bottom of the frame to enclose the bottom portion of the frame. The plate provides a surface to place the batteries as well as component protection from debris and water.

\subsection{Drive System}

Vasilius uses two 24 volt DC motors to power the two drive wheels independently. The motors are attached to the drive wheels at 90 degree angles and pivot vertically through a bracket welded to the frame. The brackets prevent any horizontal movement reducing stress on the motors. The motors are attached to the suspension system and travel with the wheels independently. The mount angles also vary as the vehicle travels across uneven ground. This ensures that a motor will not hit the ground when its respective wheel goes into a hole. The two rear wheels are free to rotate and change direction as the vehicle changes direction. The rear wheels are mounted on a pivoting arm that allows the wheels to travel vertically, independent of the main drive wheels. The pivoting arm allows 30 degrees of rear wheel travel in both directions. This differential drive system design provides the vehicle with a minimum width, short wheel base, low center of gravity, and significant ground clearance.

\subsection{Vehicle Body}

The vehicle's body frame work was constructed from 1 inch square aluminum tubing. The exterior of the body consists of formed aluminum with lexan panels around the entire surface. The entire body was light-weight, waterproof, and capable of protecting the components inside. The lexan 
panels were held in place with quarter-turn fasteners that can be quickly removed. Due to the number of panels and their positions, different components could be accessed without removing the entire enclosure. The vehicle body protects components from water, and heat as well. The outer shell was equipped with fans that cool and circulate the air inside the vehicle. Shelving inside the vehicle body allowed for component positioning and spacing assisting in cooling the interior of the structure. The vehicle body design achieved good component protection, maximum space utilization, ease of maintenance, as well as a "stealthy" appearance.

\section{Electrical System}

The goal of the Vasilius team was to model an electrical system to support the human decisionmaking process. This idea was new to the team and more complex than the system within any of the previous BSC vehicles. However, the team adequately achieved these goals and objectives in the design, producing a new and improved electrical system capable of winning the IGVC. The electrical system consisted of four parts; the power system, sensors, computers, and vehicle control.

\subsection{Power System}

Two 12-volt deep-cycle marine batteries were connected in series to provide the power to the controller, motors, main computer, and Laser Measurement System. Two smaller 12V batteries power the sensors, emergency stop contactor, and a DC-DC converter. The on-board laptop was equipped with two batteries for its own power. The DC$\mathrm{DC}$ converter provides $+12 \mathrm{~V},-12 \mathrm{~V}$, and $5 \mathrm{~V}$ for the various requirements of the electronics. After performing a power

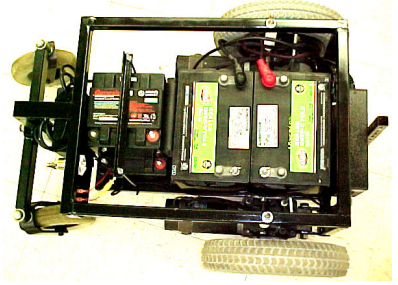
consumption analysis, the team was able to balance the power consumption across all of the batteries. This balance provided maximum run time and prevented "weak links" in the power system. In normal operation, the vehicle operated for 6 hours on a fully charged set of batteries. All of the batteries were mounted and connected with quick replacement in mind. A complete replacement of batteries could be completed in just a few minutes.

Vasilius was equipped with its own on-board charging system. The charging system consisted of one $24 \mathrm{~V}$ charger and two $12 \mathrm{~V}$ chargers. The on-board laptop also had its own charger. Once switched to a charging mode, all batteries and electronics were isolated. The electronics were then available to be powered from an AC outlet. Therefore, all the batteries were charged simultaneously, and the vehicle was tested via an extension cord while the batteries were charging. Vasilius had battery monitors on-board as well. The LED displays allow the user to see the voltage level of every battery, so that batteries can be changed out or charging could be initiated if voltage levels became low.

\subsection{Sensors}

Vasilius was equipped with a multitude of sensing devices. This variety of sensors was chosen in the electrical system design to provide various levels of data and redundancy similar to human senses. The following were sensors on-board Vasilius, a brief summary, and their respective data have been included below: 
- Stereoscopic Camera - The team designed and built a stereoscopic camera using two grayscale board-level CCD cameras with $6 \mathrm{~mm}$ lenses. The two cameras had to be synchronized, integrated, and packaged. The stereo camera mimics human eyes. Somewhat like the human brain, Vasilius could take two slightly different images and create one image with depth information. The ability to associate distance with objects using only cameras was extremely valuable in the sensor fusion process. Camera data contains the entire environment: lines, potholes, obstacles, etc.

- LMS - The Laser Measurement System (LMS) used a laser to scan 180 degrees of the environment the vehicle is traveling towards. The LMS data contained the precise distance and angle of all obstructions in its field of view.

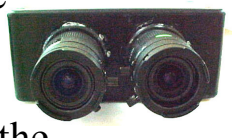

DGPS - The Differential Global Positioning System (DGPS) used the global positioning satellites to obtain a position fix. It then used a reference station or Wide Area Augmentation System (WAAS) satellites to obtain corrections that improved position accuracy. The DGPS data contains position (latitude, longitude), heading, and velocity.

- Digital Compass - The digital compass detected the earth's magnetic fields. The digital compass data contained accurate heading data, even when moving slow or stationary.
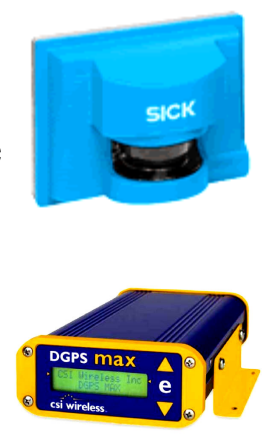
heading data, even when moving slow or stationary.

- Encoders - The encoders detected movement of the motor shaft with good precision. These encoders were also capable of measuring ambient temperature. The data from the encoders contained position, velocity, azimuth, and motor temperature.
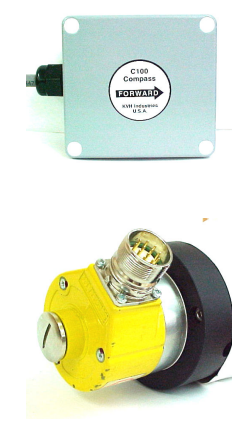

- Diffuse Sensors - Diffuse sensors detected a user defined color. By emitting light that reflected from a surface back to the sensor, the frequency can be analyzed and compared to a programmed frequency. The sensors could be programmed to detect a particular frequency (color) on the ground.

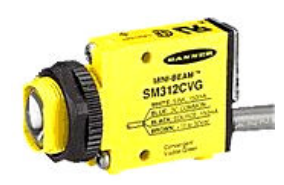

- Photoelectric Sensors - Photo (Proximity) Sensors detected obstructions. By emitting light that reflected from a surface back to the sensor, an obstruction could be found.

After selecting the sensors, the team categorized, prioritized, and integrated the

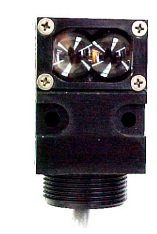
devices. The sensors were categorized as planning, reactive, or feedback devices. Next, the sensors were prioritized to achieve multiple levels of redundancy. After participating in previous competitions the team witnessed a lack of redundancy built into most ground robotic vehicles. The Vasilius sensor design focused on providing the vehicle with human-like redundancy having sensors that "back up" other sensors. In basic human navigation a plan is devised and then 
executed. However, if some unforeseen situation occurs, a reaction must occur in real time and a new plan implemented. Vasilius mimics this method by using planning sensors to devise a continually planned path of navigation. Isolated from the planning process, reactive sensors constantly check for mistakes and trapping situations. The two processes were performed in parallel by interacting and trading control when necessary. Feedback was provided for both processes and constantly updated. This approach provides more opportunities to correct mistakes and identify traps/dead ends. Most importantly, the vehicle was safe to be around. By design, it should not run off the course or crash into an obstacle. Table 2 pairs the sensor categorization.

\begin{tabular}{|l|l|}
\hline Device & Category \\
\hline Stereoscopic Camera & Planning \\
\hline LMS & Planning \\
\hline DGPS & Planning/Feedback \\
\hline Encoders & Feedback \\
\hline Digital Compass & Reactive/Feedback \\
\hline Diffused Sensors & Reactive \\
\hline Photo Sensors & Reactive \\
\hline
\end{tabular}

Table 2: Sensor Categorization

\subsection{Computers}

Designing an electrical system modeled after human decision-making required a great deal of processing power. The human brain is divided into many sections that are responsible for different thinking processes. These processes occur simultaneously, yet separate from one another. However, the processes communicate and update each other constantly. The team was interested in two of the processes: planning and reaction. The computing system was divided into two parallel systems to achieve this idea. A central computer is responsible for planning and vehicle control, while a second computer constantly checks for unforeseen situations and the correct execution of the desired plan. Working together, the computers can provide a redundant, effective, and self-monitoring means of navigation. A third, off-board, computer was also implemented to provide remote control, monitoring, and convenience. The three computers are listed below.

\section{1). Onboard Central Computer (primary)}

- Specifications:

○ $533 \mathrm{MHz}$ processor

- 128 MB RAM

- Windows 2000

○ 4-Input frame grabber with two 2-input synchronization capability 
- Responsibilities (Planning)

- Controls motors and brakes for vehicle control.

- Responsible for camera inputs, image processing and image analysis.

- Receives peripheral updates from secondary computer.

- Makes navigational decisions based on the vision algorithm.

- Plans based on data from secondary computer.

- Feedback Role

- Positioning - Encoders.

- Speed - Encoders

- Azimuth - Encoders.

- Integration Devices:

\begin{tabular}{|c|c|}
\hline Device & $\begin{array}{c}\text { Connection } \\
\text { Type }\end{array}$ \\
\hline Stereoscopic Camera & Frame grabber \\
\hline Laptop (on-board) & Serial \\
\hline Motors Controller & Parallel \\
\hline Encoder & Serial \\
\hline $\begin{array}{c}\text { Wireless Network } \\
\text { Antenna }\end{array}$ & USB \\
\hline
\end{tabular}

Table 3: Integration Devices: Onboard Central Computer

2). Onboard Laptop (secondary)

- Specifications:

- $1.8 \mathrm{GHz}$ processor

- 256 MB RAM

$\circ$ Windows XP

- Responsibilities (Reactive)

- To obtain and interpret/analyze all non-vision peripheral data.

- Make decisions based on data.

- Relay information to the central computer.

- Feedback Role

○ Positioning - GPS.

○ Heading - Digital Compass 
- Integration Devices:

\begin{tabular}{|l|l|}
\hline \multicolumn{1}{|c|}{ Device } & \multicolumn{1}{c|}{ Connection Type } \\
\hline LMS 220 & $\begin{array}{l}\text { PCMCIA 500buad high-speed } \\
\text { card }\end{array}$ \\
\hline Central Computer & Serial \\
\hline GPS Receiver & Serial \\
\hline Diffuse Sensors & Parallel \\
\hline Photo Sensors & Parallel \\
\hline Strobe Light & Parallel \\
\hline Digital Compass & Serial \\
\hline
\end{tabular}

Table 4: Integration Devices

3.) Off-board laptop (Monitoring and Remote)

- Specifications:

- $1.8 \mathrm{GHz}$ processor

- 256 MB RAM

$\circ$ Windows XP

- Responsibilities

- To provide a convenient method of remote control.

- Perform system monitoring during testing.

- Allows user to see the following data:

$>$ Acquired images.

$>$ Sensor status.

Algorithmic computations.

- System monitoring without interrupting vehicle operation.

- Gives user a valuable tool for testing and debugging

- Allows user to do the following:

$>$ Write new algorithms.

$>$ Modify/Make changes to existing algorithms.

$>$ Execute software on the vehicle's main computer remotely.

- Feedback Role

- Overall systems operation.

- Status

- Accuracy

- Consistency 
- Integration:

\begin{tabular}{|l|l|}
\hline \multicolumn{1}{|c|}{ Device } & \multicolumn{1}{c|}{ Connection Type } \\
\hline Wireless & PCMCIA wireless \\
Networking & card \\
\hline
\end{tabular}

Table 5: Integration

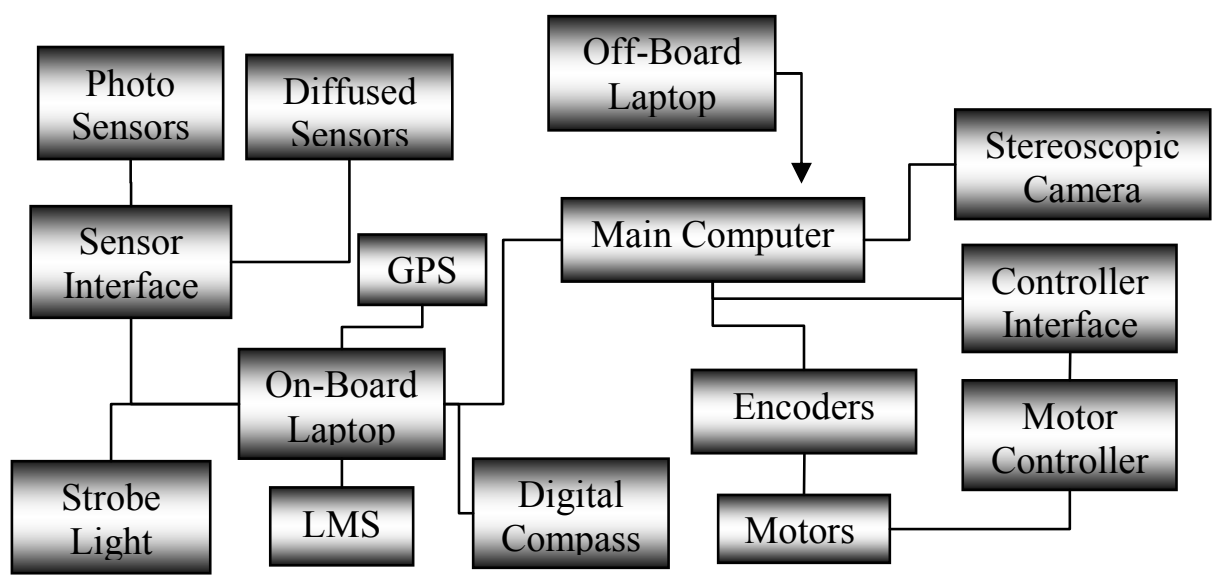

\subsection{Vehicle Control}

Vasilius utilized a closed-loop proportional integrated derivative control system consisting of a central computer, controller interface, motor controller, motors, and encoders. The used motor controller originated from an electric wheelchair. Therefore, it required an analog signal from a joystick. The team designed and built an interface that provided the analog outputs from the computers digital signal. Two analog signals were generated, one for forward/reverse and one for left/right. The multi-axis controller then sent the correct signals to the motors. Encoders monitored the motors and provided feedback to the central computer. The electric wheelchair motor controller was chosen because it was designed for a signal human input that controls vehicle direction, speed, and azimuth. This method of control enhances our human-like overall design.

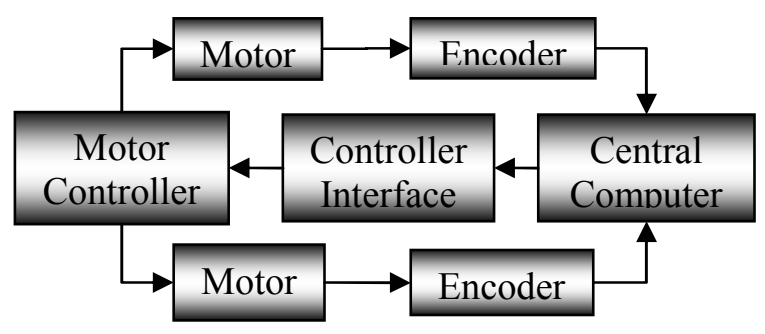




\subsection{Safety}

Safety was the most important concern in all aspects of design, fabrication, and operation of the robot. We think that part of its autonomous function was safety. This was accomplished through many different processes and was infiltrated throughout the design process. Especially important were the two manual pushbuttons located at the rear of the GRV which when pressed disconnected power to the motors thereby effectively stopping the robot. In addition, a remote estop system was included consisting of an infrared transmitter and a receiver circuit board equipped with two relays. Fuses added at each battery protected against damaging power surges.

The mechanical design of the vehicle offered additional safety in that it could swing out of difficult positions because it was differentially steered. Our overall system of interrelating sensors provided us the opportunity for safe navigation because of the inherent redundancy applied throughout our software.

\section{Software Design/Autonomous Algorithm}

\subsection{Design Objectives}

As stated in the design process and in the competition requirements, design objectives for our software design were:

- To develop an autonomous algorithm that processed images and all long-range planning computations at least once per second and all short-range reaction computations at least once per 10 milliseconds. The "one second" and "10 millisecond" specifications were based on robot speed and size.

- To develop a software structure that could be easily maintained and accessed by many different programmers.

- To write the most efficient program possible for performing all four major autonomous functions and testing.

- To keep safety, reliability, and durability top priorities in software design

In order to meet these software design objectives we had to choose and optimally integrate the sensor suite of the robot. First, we needed to describe this sensor integration.

\subsection{Sensor Integration}

The sensors on Vasilius fused together to provide a broad range of sensory input in order for the vehicle to perform four major functions; lane following, object detection, leader following, and waypoint navigation. The team developed sensor integration on Vasilius using two complimentary ideas: long-range trajectory planning and short-range reaction.

\subsection{Sensor Functions}

The robot sensed its trajectory twice per second using any combination of the three planning sensors. If no errors occurred in trajectory planning and execution, the vehicle did not need to use the reaction sensors. In the event a reaction sensor detected a road edge or close obstacle, the vehicle responded to the reaction sensor. Once the reaction sensors were all clear, then the 
vehicle would go back to trajectory planning. Testing had shown this concept worked quite well. Many times during testing we had to force errors in the planning process to test the reaction process.

This philosophy was again similar to human navigation. Just as humans use priority levels in their senses, the robot used a priority system for the sensors. Although the stereo camera and DGPS priorities are low, these sensors were used all the time except during "interrupts" from the reaction sensors.

Vasilius' algorithm combined the information gathered from the LMS and the stereoscopic camera to produce a 3-D map of the course in front of the robot. This map illustrated lanes and obstacles up to eight meters away. The program transferred object distances measured by the LMS to the map produced from the stereoscopic images. A description of the robot employing these concepts and sensor integration to perform the four major functions is outlined below:

\subsection{Lane Following and Obstacle Avoidance}

Vasilius used a trajectory planning process to plot the optimal path between lanes and obstacles using the 3-D map produced from the fusion of stereoscopic images and LMS data. The processor used several methods of image processing: Sobel Amplitude and Canny edge detection, as well as Binary Thresholding. The processing speed of the robot was very important, therefore, instead of analyzing each pixel of the map, we developed two other timesaving approaches named the "nine-line algorithm" and the "push algorithm". The "nine-line algorithm" determined the path that had the widest room between lanes and obstacles on each of the nine equally-spaced horizontal lines (this divided the map into ten equally-sized horizontal slices.) Given the camera angle and field of view, the distance between two consecutive lines was slightly smaller than the white pail, the smallest obstacle on the course. The algorithm then established candidate paths with the most "elbow" room between each horizontal line. Both maximum and minimum distances from obstacles and lanes were allowed since one lane may have been absent or dashed. The robot turn angle and time of turn were a combination of past and present paths that created a recursive-type algorithm. The "push algorithm" divided the map into ten regions on the left and ten regions on the right. The sum of all pixels gray-scaled values in each region created an obstacle/line "force" that pushed the robot away from the region. Regions at the bottom of the map had more weight than regions at the top. All these "forces" were summed and the robot would take the least resistant path between them with a turn angle proportional to the resultant force.

\subsection{Leader Following}

The leader following algorithm operated in almost an inverted method as compared to lane following/obstacle avoidance. Instead of avoiding lanes and obstacles, this algorithm tracked an object. Using Object Detection and LMS data, we turned the robot left and right so as to keep the object in the center of the vision screen and at a specified distance. The robot maintained distance using a combination of stereo vision and LMS data. If the disparity of the two detected objects from the stereo images decreased, the robot increased speed, and vice versa. LMS 
measurements also provided distance. The LMS was only used to ensure that the object being tracked stayed within a specific distance threshold.

\subsection{Waypoint Navigation}

Vasilius' waypoint navigation algorithm used DGPS, a digital compass, LMS, and proximity sensors to navigate around obstacles to target points. This algorithm had the structure that follows:

1) Input coordinates of Start and all Targets

2) Find initial error between given and measured Start position

3) Start Vasilius in forward motion

4) Move to next target by comparing robot position to next target position

5) Monitor LMS and proximity sensors for obstacles

6) If obstacle, react until clear, go to Step 4

7) Increase target number once target is found

8) If target number is ten, then stop robot

9) If target number $<$ ten, go to Step 4

The DGPS receiver had both WAAS-enabled and differential beacon modes. With a beacon, we could achieve 2 -foot accuracy $67 \%$ of the time. By subtracting initial errors between given and measured positions at the start, we could subtract off this error. As long as the robot could finish the course in less than five minutes, DGPS errors did not change significantly.

\section{Analysis of Predicted Performance and Results}

Design and testing indicates that Vasilius would perform as indicated in the table below. The table also indicated the results of our testing. A "** symbol means that we have not yet measured that performance item. Each prediction listed in the table comes from analyses of components as well as overall performance.

\begin{tabular}{|l|l|l|}
\hline Performance Measure & Performance Prediction & Performance Results \\
\hline Speed & $5 \mathrm{mph}$ & $4.9 \mathrm{mph}$ \\
\hline Ramp Climbing & 20 -degree incline & 18 -degree incline \\
\hline Turn Reaction Time & 360 degrees/ second & 315 degrees/ second \\
\hline Battery Life & 8 hours weakest link & 6 hours weakest link \\
\hline Stop Reaction Time & Immediate & Almost Immediate \\
\hline Object Detection & 0 inches to 32 feet & 2 inches to 30 feet \\
\hline Dead-Ends and Traps & Chosen paths are clear & $*$ \\
\hline Potholes & Chosen paths are clear & $*$ \\
\hline Waypoint Accuracy & 2 feet one sigma & 2 feet one sigma \\
\hline
\end{tabular}




\section{Other Design Considerations}

\subsection{Safety}

Safety was again our most important concern in all aspects of the design, fabrication, and operation of the robot. Safety would be part of all autonomous design function. This was accomplished through many different processes and was infiltrated throughout our design process. Especially important were the two manual pushbuttons located at the rear of the vehicle which, when pressed, disconnect power to the motors thereby effectively stopping the vehicle. In addition, a remote e-stop system was included consisting of an infrared transmitter and an onboard receiver. Fuses, circuit breakers, and disconnect switches protect all of the robotic components from overloads, noise spikes, and short circuits. The overall system of interrelating sensors provided the team an opportunity for safe navigation because of the inherent redundancy applied throughout our software.

\subsection{Reliability}

Past teams observed a noted lack of reliability in many of the vehicles from past competitions. Therefore, the reliability of this system was stressed to the team. Redundancy was emphasized as a design tool to achieve a higher level of reliability. As described in Software Design, different sensor groups had redundant functions. Both the stereo camera and the diffused sensors detect the presence of lanes. The stereo camera algorithm also doubled with the LMS and proximity sensors in detecting objects.

\subsection{Durability}

Solid mechanical design made the vehicle very durable. Its framework housed and protected components. The exterior shell of the vehicle prevented water and debris from coming in contact with the electrical system. Components on the exterior of the vehicle were waterproofed and designed to withstand minimal damage. The vehicle could be operated under normal circumstances without great concern for accidental damage to vital components or affecting the vehicle's overall performance.

\subsection{Battery Life}

Four twelve volt batteries were used to power all electrical devices contained on the robot. The GRV team designed a battery charger and meter so that our batteries could be charged without the timely process of taking the cover off. Tests demonstrated that our batteries could last for a continual operation of around 5 hours. The laptop battery lasted approximately 2 hours.

\subsection{Obstacles Detection}

An important factor in any autonomous navigation is the successful detection of obstacles in and around the vicinity of the moving robot. Stereoscopic cameras enabled the robot to critically maneuver around objects such as potholes and barrels. Longer range objects up to around $75 \mathrm{ft}$. could be detected by the laser range finder with good precision. The closest objects missed by the cameras were then detected by 6 diffuse sensors and 6 proximity sensors that were located on the outer frame. 


\subsection{Dead Ends, Traps, and Pot Holes}

Finally, the robot had the capability of recognizing traps and dead ends upon approach through the action of our sensors which communicate with the laptop. The diffuse sensors could be set for certain colors so that sand-traps could be avoided. The robot avoided constraining traps because of the attention to detail in the design phase.

\section{Senior Design Course}

The important side benefit to implementing this applied research robotics project in the ELET 492 Senior Design course, where student teams build robotics for our defense agency clients, was realized with their success in the annual International Ground Robotics Vehicle Competition. This program became a definite benefit to motivating our students as well as satisfying our clients.

ELET 492 is a two credit hour course with a one hour meeting and three laboratory hours each week to include the design and fabrication of a project in the field of engineering technology under the supervision of an instructor, culminating in a written report and an oral defense of the selected project before a select faculty committee and other invited guests. Successful projects have resulted in journal articles or may be entered into contests.

The instructor of record was Dr. Robert N. Riggins, but a project advisor could be any instructor in the ELET Department (not necessarily the ELET 492 course instructor). It was the student's job to find a faculty member willing to act as project advisor to the student's project. The responsibilities of the project advisor include the following:

(1) Provide technical guidance throughout the duration of the project

(2) Maintain contact by meeting with the student(s) at least once a week to monitor progress

(3) Order project parts if finances allow. In the case of inadequate finances, then the project advisor must help the student(s) find an alternate plan. If the project itself is terminated, then the course advisor (see below) helps the student(s) find a new project and a new project advisor.

(4) If necessary, find fund sources and parts sources, such as grants and sponsors for the project.

However, the instructor was still the faculty member assigned responsibility for the outcomes in ELET492. The responsibilities of the course instructor include the following:

(1) Meet regularly once a week with all ELET 492 students to monitor progress.

(2) Organize and grade all ELET492-related oral presentations and functions. Individual projects entering into other functions not related to ELET492 will be the responsibility of the project advisor.

(3) Collect and grade all final written project reports.

(4) Submit attendance reports and mid-semester grade reports

(5) Assign final grades (the course instructor should consult with the project advisor). 
(6) Ensure that each student taking ELET492 has a topic and a project advisor.

Note: The course instructor will most likely be project advisor for most of the projects. The prerequisite has been limited to obtaining the consent of instructor and the Dean of our School for Engineering Technology and Computer Science. (This is a senior-level course, so most of the required courses have been taken by the student.)

There was no text book for the course. Students were encouraged to reference a variety of resources including all of their other engineering texts, related articles, internet, product catalogs, and personal interviews, internships, etc.

Upon the successful completion of this course, the student had developed applied knowledge gained from all previous courses and experiences to conceive, design, fabricate, and troubleshoot a practical project in the field of electrical engineering technology. The students gained respect for and within the technical community due to the visibility and relationships resulting from this project.

\section{Objectives:}

$\mathrm{X}$ To identify a project topic that is useful and at the same time doable

$\mathrm{X} \quad$ To do a complete design such that fabrication is straightforward

$\mathrm{X}$ To build the project using off-the-shelf and available parts

$\mathrm{X} \quad$ To make the project successful via troubleshooting techniques

$\mathrm{X} \quad$ To be able to communicate the project results to the technical community

The scheduled course events and minimal requirements were as follows:

\section{Week Milestone}

$1 \quad$ Initial 1 hour meeting to set ground rules

21 page project proposals per individual due

14 20-minute-per-team final presentations to invited guests

$15 \quad$ Final individual reports due

Regarding Week 14 presentations, bigger projects took longer than 20 minutes (Everybody Talks!). In addition to the above, every week starting on the 3 rd week, each team gave the course instructor a brief 1 paragraph summary of the past week's progress and the plans for the coming week (the student can send the weekly summaries via e-mail, but no telephone calls). These weekly reports were a single paragraph and can be handwritten (however, typed reports could be saved and used in writing the final report). The above requirements are minimal, and were completed in teams.

Computer usage was an obvious mandatory requirement in preparation of the final report, since these reports were later written in publishable form.

The instructor assigned grades to the projects with the following attributes in mind: 
(1) Originality

(2) Difficulty

(3) Completeness / Accuracy

(4) Format / Communication

(5) Analysis

Material for grading came from the presentation, written report, and the timeliness and content of the weekly progress reports. The course requirements outlined above will be graded as follows:

$\begin{array}{ll}\text { Proposal } & 10 \% \\ \text { Weekly reports } & 15 \% \\ \text { Final oral presentations } & 30 \% \\ \text { Final written report } & 45 \%\end{array}$

Late items are penalized by deducting $10 \%$ per day from the score of each late item.

\section{Conclusion}

Vasilius won two world titles at the $11^{\text {th }}$ Annual International Ground Robotic Vehicles in Rochester, Michigan this past summer for Best Design and Most Intelligent Robot raising both the stature of the College and its programs to a higher level of competitiveness. The autonomous vehicle was designed, built, and manufactured by students from the Bluefield State College ELET 492 Senior Design course with funding provided by the Center for Applied Research and Technology, Inc. The initial vision for building the AGRV as outlined by the project advisor included one of safety, reliability, durability, and versatility. We think the robot embodies all of those qualities due in part to the rigorous design process that we systematically followed. This is the best testament to the successful implementation of an open ended project design and the extra effort and optimism providing inspiration to a motivated student team.

Robert N. Riggins

Robert N. Riggins is an Associate Professor of Electrical Engineering Technology at Bluefield State College. He has been teaching in the electrical department for the past 6 years. In August of 1996, he retired with the rank of Lieutenant Colonel from the United States Air Force after 20 years of service. His experience in the field of Electrical Engineering spans the last 26 years, and includes a broad range of topics such as electronics, navigation, control, avionic sensors, robotics and sensors, computer programming, microcontrollers, and microprocessors.

Bruce V. Mutter

Bruce V. Mutter, the founding director of the Center for Applied Research \& Technology, Inc. (CART), teaches project management and engineering economics at Bluefield State College as an Associate Professor in the Division of Engineering Technology. He earned his A.S. and B.S. degrees in Architectural and Civil Engineering Technology from Bluefield State College, and his M.S. degree in Construction Management and C.A.G. in Environmental Design and Planning from Virginia Tech. He is a current Ph.D. candidate at Virginia Tech and completed additional graduate coursework with the University of New Mexico and New Mexico State University. 